\title{
Exact Solution for PTT Fluid on a Vertical Moving Belt for Lift with Slip Condition
}

\author{
Syed Mansoor Shah ${ }^{1}$, K. N. Memon ${ }^{1,3^{*}}$, Syed Feroz Shah ${ }^{1}$, Abdul Hanan Sheikh ${ }^{3}$, \\ Abbas Ali Ghoto ${ }^{3}$, A. M. Siddiqui ${ }^{2}$ \\ 'Department of Basic Science, Mehran University of Engineering Technology Jamshoro, Pakistan; \\ msyed3511@gmail.com, knmemon@quest.edu.pk, feroz.shah@faculty.muet.edu.pk, \\ ah.sheikh@quest.edu.pk, abbasghoto@gmail.com \\ 2Pennsylvania State University, York Campus, Edgecombe-17403, \\ USA, Pakistan; ams5@psu.edu \\ ${ }^{3}$ Department of Mathematics and Statistics, QUEST, Nawabshah, Pakistan
}

\begin{abstract}
Objectives: This attempt is made to investigate the problem of a study of thin film flow for lift while considering a steady, incompressible, non-isothermal, Phan-Thien Tanner fluid on a vertical belt with slip condition. Method/Comparative analysis: The nonlinear differential equation has been derived from the continuity and momentum equations. Exact solution has been obtained, which gives us velocity-profile of fluid, flow rate, temperature, average velocity of fluid and net upward flow. The special cases such as the linear PTT (LPTT), quadratic PTT (QPTT), cubic PTT (CPTT), exponential PTT (EPTT) and upper convected Maxwell (UCM) models are considered, from the same model of PTT fluid. The behavior the velocity and depth is discussed, in effect of various parameters. The velocity and temperature are compared for those special cases. Findings: The analogy of the EPTT, CPTT, QPTT, LPTT and UCM fluid models for the velocity profile and temperature distribution reveals that the velocity profile of the UCM fluid model increases quickly as compared to the velocity of the EPTT fluid. The temperature distribution for EPTT fluid is higher than the temperature for its special case: UCM fluid model. The velocity of PTT fluid is observed to be increased with incorporation of slip condition on vertical belt. Improvements: None of the previous work has been done using no-slip boundary conditions, however slip conditions are used in piece of work. Also, a thorough discussion on cases is novelty in this work.
\end{abstract}

Keywords: Exact Solution, Heat Transfer, Lift Problem, Phan-Thien Tanner, Thin Film Flow

\section{Introduction}

The non-Newtonian fluids have applications in many areas including biology, chemical engineering, polymer industry. Thus it has attracted overwhelmingly attention from researchers recently. Because of its vast applicability, the non-Newtonian fluid problems are hard to be modeled using a single model (1). Thus many equations have been proposed over the time to model problems involving non-Newtonian fluids (2-5). Such models include PhanThien-Tanner fluid model, Sisco fluid model, second and third order fluid model, and Power law fluid model. Out of these, the Phan-Thein Tanner fluid model is extensively researched, because of its clarity, simplicity and various applications in many areas of engineering and industry (6-8).

For these models, the techniques to obtain exact solution are rarely discussed for the equation of motion specially for non-Newtonian fluids, because of the nonlinear nature of these equations $(9,10)$. The accuracy of numerical techniques is compromised in general and such techniques are of less use especially for fluid problem, 
as parameter tuning become more difficult. The exact solution techniques are more preferred (5,11-14), for reasons, including above, that those methods give simple dynamics of fluids, also help for validation. Also the accuracy is not compromised, leading to fair comparison with results obtained from simulations and experiments.

Here our principal concentration is investigation of thin layer flow concerning a PTT fluid with slip condition $(8,15)$. The fluid is partly bounded at side, whereas another liquid is considered at next boundary. The study of thin layer flow is significant concerning chemical processing. The flow of a films in the human eye membrane, paint on the wall and rainwater falling down the window $(6,7,16,17)$ are some real life examples.

In this manuscript, we studied thin-film flow on belt moving upwards, lifting thin layers, considering PTT fluid along with slip condition on boundary of solid wall. The exact solution of the consequential differential equation has been chalked out, while considering boundary conditions. For the slip parameter, we obtain velocity in case of linearly viscous fluid (6). Four estates are examined, namely CPTT, QPTT, LPTT and UCM. As the best of our insight the results by using perturbation method is not accounted anywhere.

This paper is structured as follows: Section 2 provides basic equation's for the PTT fluid. The formulation of the problem and solution is chalked out in Section 3. Section 4 is dedicated to Results and discussion and conclusions are drawn in Section 5.

\section{Basic Equations}

The governing equations for incompressible PTT fluid including thermal effects are (5)

$$
\begin{aligned}
& \nabla \cdot \mathbf{V}=0 . \\
& \rho \frac{D \mathbf{V}}{D t}=-\nabla p+\rho \mathbf{f}+\nabla \cdot \mathbf{S}, \\
& \rho C_{p} \frac{D \theta}{D t}=k \nabla^{2} \theta+\frac{1}{2} \operatorname{tr}\left(\mathbf{S} \mathbf{A}_{1}\right)
\end{aligned}
$$

The symbol V represent velocity vector, $\rho$ constant density, $p$ dynamic pressure, $b$ body force, $S$ extra stress tensor, $\eta$ is the constant viscosity, $\theta$ is the temperature, $C p$ is specific heat constant, $k$ is thermal conductivity. The operator $\frac{D}{D t}$ denotes the material derivative and $A_{1}$ be the $1^{\text {st }}$ Rivlin Ericksen tensor, which is

$$
\mathbf{A}_{1}=\nabla \mathbf{V}+(\nabla \mathbf{V})^{T}
$$

The equation of an incompressible PTT fluid model $(6,8)$ can be read as

$$
f(t r \mathbf{S}) \mathbf{S}+\lambda \stackrel{\nabla}{\mathbf{S}}=\eta \mathbf{A}_{1}
$$

Here $\lambda$ is the relaxation time and $\stackrel{\vee}{s}$ be the upper convected derivative which is

$$
\stackrel{\nabla}{\mathbf{S}}=\frac{D \mathbf{S}}{D t}-\left((\nabla \mathbf{V})^{t} \mathbf{S}+\mathbf{S}(\nabla \mathbf{V})\right)
$$

Five PTT models, frequently used are

1. UCM Modell $1 \quad f(t r S)=1$

2. LPTT Model $\quad f(t r S)=1+\frac{\varepsilon \lambda}{\eta} \operatorname{tr} \mathbf{S}$

3. QPTT Model

$$
f(t r S)=1+\frac{\varepsilon \lambda}{\eta} t r S+\frac{\delta_{1}}{2}\left(\frac{\varepsilon \lambda}{\eta} t r S\right)^{2}
$$

4. CPTT Model

$$
f(t r \mathbf{S})=1+\frac{\varepsilon \lambda}{\eta} t r \mathbf{S}+\frac{\delta_{1}}{2}\left(\frac{\varepsilon \lambda}{\eta} \operatorname{tr} \mathbf{S}\right)^{2}+\frac{\delta_{2}}{6}\left(\frac{\varepsilon \lambda}{\eta} \operatorname{tr} \mathbf{S}\right)^{3}
$$

5. EPTT Model

$$
f(t r S)=\exp \left(\frac{\varepsilon \lambda}{\eta} t r S\right)
$$

Where $\varepsilon$ is elongational behavior and $\delta_{1} \delta_{2}$ are parameters for QPTT and CPTT. Higher the value of parameter $\varepsilon$, the thinner the fluid is. In other words, the value of $\varepsilon$ defines the shear thinning effect. In such case, flow problem is modeled using Phan-Thien Tanner flow model. Further the exponential PTT is specified for thinner fluid, than in case of linear PTT. Also the $\varepsilon$ is inversely proportional to elongational viscosity $\underline{\underline{6}-\underline{8} \text {. }}$. 


\section{Formulation of the Problem and Solution}

Consider a belt, of substantial width, is moving vertically in a container filled in PTT fluid, against gravitational force in the container with constant velocity $U$, as illustrated in Figure 1. While moving, a film of fluid is moving alongwith belt. The $\delta$ is thickness of the film, which uniform. Further assumptions are; the flow is steady, laminar and parallel under atmospheric pressure $p$. Accordingly we assume that

$$
\mathbf{V}=[0, v(x), 0] \quad \mathbf{S}=\mathbf{S}(x) \quad \theta=\theta(x)
$$

The corresponding free space and slip boundary conditions are:

$$
\mathrm{S}_{x y}=0 \text { and }=0 \text { at } x=\delta \text { Free space }
$$
condition

$\mathrm{v}=\mathrm{U}-\left.\beta \mathrm{S}_{x y}\right|_{x=0}$ and $\theta=\theta_{0} \quad$ at $x=0$ Slip boundary condition

It is permanent to note that the equation (12) satisfies continuity equation (1). Further, incorporation of equation (12) into equation (2) gives the non-zero momentum at atmospheric pressure

$$
0=-\rho g+\frac{d S_{x y}}{d x}
$$

and equation (3) leads to energy equation, with incorporation of equation (12),

$$
0=k \frac{d^{2} \theta}{d x^{2}}+S_{x y} \frac{d v}{d x} .
$$

Also the value of first Rivlin Ericksen tensor is used in equation (16).

Integrating equation (15) with respect $\mathrm{x}$ and incorporating boundary conditions from equation (13), it leads to equation

$$
\mathrm{S}_{x y}=\rho g(x-\delta)
$$

Inserting equation (12) into the equations (4)-(6), after considerable calculations once we obtain:

$$
f(t r \mathbf{S}) \mathrm{S}_{x x}=f(t r \mathbf{S}) \mathrm{S}_{z z}=f(t r \mathbf{S}) \mathrm{S}_{\mathrm{zx}}=0
$$

$$
\begin{aligned}
& f(t r \mathbf{S}) S_{y z}=S_{z x} \frac{d v}{d x} \\
& f(t r \mathbf{S}) S_{x y}=\eta \frac{d v}{d x}+\lambda S_{x x} \frac{d v}{d x} \\
& f(t r \mathbf{S}) S_{y y}-2 \lambda S_{y x} \frac{d v}{d x}=0
\end{aligned}
$$

Since $f(t r \mathbf{S})$ has one of the values given in equation (7) $-(11)$, therefore. $f(\operatorname{tr} \mathbf{S}) \neq 0$ Which implies that

$$
\mathrm{S}_{x x}=\mathrm{S}_{z z}=\mathrm{S}_{z x}=0
$$

By applying these values from equation (22) into the equations (19)-(21) we get

$$
\mathrm{S}_{y z}=0
$$

$$
f(t r \mathbf{S}) S_{x y}=\eta \frac{d v}{d x}
$$

$$
f(t r \mathbf{S}) S_{y y}=2 \lambda S_{y x} \frac{d v}{d x}
$$

Joining equations (24) and (25), we get

$$
S_{y y}=\frac{2 \lambda S_{x y}^{2}}{\eta}
$$

The shear and normal stresses are related in Eq. 26.

After applying equations (22) and (26), the trace of extra stress tensor is given as

$$
t r \mathbf{S}=\frac{2 \lambda S_{x y}^{2}}{\eta}
$$

The normal stress is obtained from equation (26), while using sheer stress from equation (17), which is given as

$$
S_{y y}=\frac{2 \lambda \rho^{2} g^{2}(\delta-x)^{2}}{\eta}
$$

equation (24) can be rewritten as

$$
\frac{d v}{d x}=-\frac{\rho g f(\operatorname{tr} \mathbf{S})(\delta-x)}{\eta}
$$


Exact solutions of this equation along with the boundary conditions (13)-(14) are obtained for five cases (7)-(11) in the following sections:

\subsection{Solutions of UCM Model}

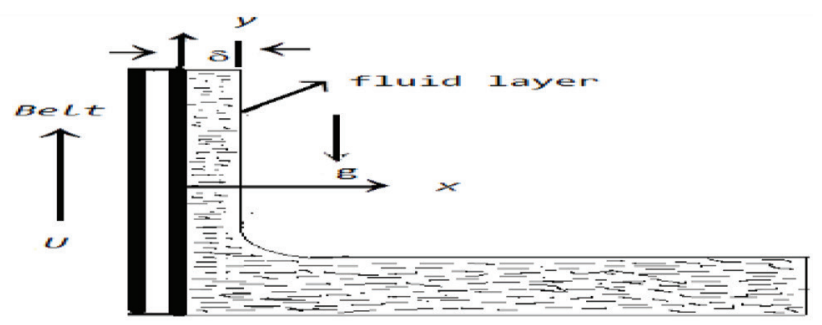

Figure 1. Illustration of the vertical moving belt through PTT fluid.

\section{Velocity Profile}

Using UCM model, which is given in (7), in equation (29) and then applying equation (14), we get

$$
v(x)=U+\beta \rho g \delta-\frac{\rho g}{2 \eta}\left(2 \delta x-x^{2}\right)
$$

Flow Rate

The $\mathrm{Q}$ denotes the flow rate between 0 to $\delta$, which is

$$
Q=\int_{0}^{\delta} v(x) d x
$$

Using equation (30), we get

$$
Q=U \delta+\beta \rho g \delta^{2}-\frac{\rho g \delta^{3}}{3 \eta}
$$

\section{Average Velocity}

The equation (33) is average velocity;

$$
\begin{aligned}
& \bar{V}=\frac{Q}{\delta} \\
& \bar{V}=U+\beta \rho g \delta-\frac{\rho g \delta^{2}}{3 \eta}
\end{aligned}
$$

\section{Net upward flow}

The net upward flow of the fluid described as $\bar{V}>0$, hence net upward for equation (34)

$$
U>-\beta \rho g \delta+\frac{\rho g \delta^{2}}{3 \eta}
$$

\section{Energy equation}

By substituting equations (17) and (30) into the equation (16), hence solution of equation (16) after applying boundary conditions (13) and (14) is,

$$
\theta=\theta_{0}+\frac{\rho^{2} g^{2}}{12 k \eta}\left(\delta^{4}-(\delta-x)^{4}\right)
$$

\subsection{Solutions for Linear PTT (LPTT) Model}

In the same way as in Section 3.1, the solutions for velocity profile, average velocity, net upward flow and energy equation for LPTT model (8), as follows:

$$
\begin{gathered}
v=U+\beta \rho g \delta-\frac{\rho g\left(\delta^{2}-(\delta-x)^{2}\right)}{2 \eta}-\frac{\varepsilon \lambda^{2} \rho^{3} g^{3}\left(\delta^{4}-(\delta-x)^{4}\right)}{2 \eta^{3}} \\
Q=U \delta+\beta \rho g \delta^{2}-\frac{\rho g \delta^{3}}{3 \eta}-\frac{2 \varepsilon \lambda^{2} \rho^{3} g^{3} \delta^{5}}{5 \eta^{3}} \\
\bar{V}=U+\beta \rho g \delta-\frac{\rho g \delta^{2}}{3 \eta}-\frac{2 \varepsilon \lambda^{2} \rho^{3} g^{3} \delta^{4}}{5 \eta^{3}}
\end{gathered}
$$

$$
U>-\beta \rho g \delta+\frac{\rho g \delta^{2}}{3 \eta}+\frac{2 \varepsilon \lambda^{2} \rho^{3} g^{3} \delta^{4}}{5 \eta^{3}}
$$

$$
\theta=\theta_{0}+\frac{\rho^{2} g^{2}}{12 k \eta}\left(\delta^{4}-(\delta-x)^{4}\right)+\frac{\varepsilon \lambda^{2} \rho^{4} g^{4}}{15 k \eta^{3}}\left(\delta^{6}-(\delta-x)^{6}\right)
$$

\section{Solutions for Quadratic PTT (QPTT) Model}

Now by substituting the value of QPTT model (9), all the required solutions are:

$$
\begin{aligned}
& v=U+\beta \rho g \delta-\frac{\rho g\left(\delta^{2}-(\delta-x)^{2}\right)}{2 \eta}- \\
& \frac{\varepsilon \lambda^{2} \rho^{3} g^{3}\left(\delta^{4}-(\delta-x)^{4}\right)}{2 \eta^{3}}- \\
& \frac{\delta_{1} \varepsilon^{2} \lambda^{4}}{3 \eta^{5}} \rho^{5} g^{5}\left(\delta^{6}-(\delta-x)^{6}\right)
\end{aligned}
$$

$$
Q=U \delta+\beta \rho g \delta^{2}-\frac{\rho g \delta^{3}}{3 \eta}-\frac{2 \varepsilon \lambda^{2} \rho^{3} g^{3} \delta^{5}}{5 \eta^{3}}-\frac{2 \delta_{1} \varepsilon^{2} \lambda^{4} \rho^{5} g^{5} \delta^{7}}{7 \eta^{5}}
$$


$\bar{V}=U+\beta \rho g \delta-\frac{\rho g \delta^{2}}{3 \eta}-\frac{2 \varepsilon \lambda^{2} \rho^{3} g^{3} \delta^{4}}{5 \eta^{3}}-\frac{2 \delta_{1} \varepsilon^{2} \lambda^{4} \rho^{5} g^{5} \delta^{6}}{7 \eta^{5}}$

$U>-\beta \rho g \delta+\frac{\rho g \delta^{2}}{3 \eta}+\frac{2 \varepsilon \lambda^{2} \rho^{3} g^{3} \delta^{4}}{5 \eta^{3}}+\frac{2 \delta_{1} \varepsilon^{2} \lambda^{4} \rho^{5} g^{5} \delta^{6}}{7 \eta^{5}}$

$\theta=\theta_{0}+\frac{\rho^{2} g^{2}}{12 k \eta}\left(\delta^{4}-(\delta-x)^{4}\right)+$

$\frac{\varepsilon \lambda^{2} \rho^{4} g^{4}}{15 k \eta^{3}}\left(\delta^{6}-(\delta-x)^{6}\right)+$

$\frac{\delta_{1} \varepsilon^{2} \lambda^{4} \rho^{6} g^{6}}{28 k \eta^{5}}\left(\delta^{8}-(\delta-x)^{8}\right)$

\subsection{Solutions for Cubic PTT (CPTT)}

In the same manner substituting the value of CPTT model (10), hence the required solutions are:

$$
\begin{aligned}
& v=U+\beta \rho g \delta-\frac{\rho g\left(\delta^{2}-(\delta-x)^{2}\right)}{2 \eta}-\frac{\varepsilon \lambda^{2} \rho^{3} g^{3}\left(\delta^{4}-(\delta-x)^{4}\right)}{2 \eta^{3}} \\
& -\frac{\delta_{1} \varepsilon^{2} \lambda^{4} \rho^{5} g^{5}}{3 \eta^{5}}\left(\delta^{6}-(\delta-x)^{6}\right)-\frac{\delta_{2} \varepsilon^{3} \lambda^{6} \rho^{7} g^{7}}{12 \eta^{7}}\left(\delta^{8}-(\delta-x)^{8}\right) \\
& Q=U \delta+\beta \rho g \delta^{2}-\frac{\rho g \delta^{3}}{3 \eta}- \\
& \frac{2 \varepsilon \lambda^{2} \rho^{3} g^{3} \delta^{5}}{5 \eta^{3}}-\frac{2 \delta_{1} \varepsilon^{2} \lambda^{4} \rho^{5} g^{5} \delta^{7}}{7 \eta^{5}} \\
& -\frac{2 \delta_{4} \varepsilon^{3} \lambda^{6} \rho^{7} g^{7} \delta^{9}}{27 \eta^{7}} \\
& \bar{V}=U+\beta \rho g \delta-\frac{\rho g \delta^{2}}{3 \eta}- \\
& \frac{2 \varepsilon \lambda^{2} \rho^{3} g^{3} \delta^{4}}{5 \eta^{3}}- \\
& \frac{2 \delta_{1} \varepsilon^{2} \lambda^{4} \rho^{5} g^{5} \delta^{6}}{7 \eta^{5}} \\
& -\frac{2 \delta_{4} \varepsilon^{3} \lambda^{6} \rho^{7} g^{7} \delta^{8}}{27 \eta^{7}} \\
& U>-\beta \rho g \delta+\frac{\rho g \delta^{2}}{3 \eta}+ \\
& \frac{2 \varepsilon \lambda^{2} \rho^{3} g^{3} \delta^{4}}{5 \eta^{3}}+\frac{2 \delta_{1} \varepsilon^{2} \lambda^{4} \rho^{5} g^{5} \delta^{6}}{7 \eta^{5}} \\
& +\frac{2 \delta_{4} \varepsilon^{3} \lambda^{6} \rho^{7} g^{7} \delta^{8}}{27 \eta^{7}}
\end{aligned}
$$

$$
\begin{aligned}
& \theta=\theta_{0}+\frac{\rho^{2} g^{2}}{12 k \eta}\left(\delta^{4}-(\delta-x)^{4}\right) \\
& +\frac{\varepsilon \lambda^{2} \rho^{4} g^{4}}{15 k \eta^{3}}\left(\delta^{6}-(\delta-x)^{6}\right) \\
& +\frac{\delta_{1} \varepsilon^{2} \lambda^{4} \rho^{6} g^{6}}{28 k \eta^{5}}\left(\delta^{8}-(\delta-x)^{8}\right) \\
& +\frac{2 \delta_{2} \varepsilon^{3} \lambda^{6} \rho^{8} g^{8}}{135 k \eta^{7}}\left(\delta^{10}-(\delta-x)^{10}\right)
\end{aligned}
$$

\subsection{Solutions for Exponential PTT Model (EPTT Model)}

Finally replacing the value of QPTT model (11), all the required solutions are given

$$
\begin{aligned}
v= & U+\beta \rho g \delta-\frac{\eta}{4 \varepsilon \rho g \lambda^{2}}\left\{e^{\left(\frac{2 \varepsilon \lambda^{2} \rho^{2} g^{2}}{\eta^{2}}\right)}-e^{\left(\frac{2 \varepsilon \lambda^{2} \rho^{2} g^{2}(\delta-x)^{2}}{\eta^{2}}\right)}\right\} \\
Q & =U \delta+\beta \rho g \delta^{2}-\frac{\eta \delta}{4 \rho g \varepsilon \lambda^{2}} e^{\left(\frac{2 \varepsilon \lambda^{2} \rho^{2} g^{2} \delta^{2}}{\eta^{2}}\right)} \\
& +\frac{\sqrt{2 \pi} \eta^{2}}{16 \varepsilon^{\frac{3}{2}} \lambda^{3} \rho^{2} g^{2}} \operatorname{erfi}\left(\frac{\sqrt{2 \varepsilon} \rho g \delta \lambda}{\eta}\right)
\end{aligned}
$$

$$
\begin{aligned}
& \bar{V}=U+\beta \rho g \delta-\frac{\eta}{4 \rho g \varepsilon \lambda^{2}} e^{\left(\frac{2 \varepsilon \lambda^{2} \rho^{2} g^{2} \delta^{2}}{\eta^{2}}\right)} \\
& +\frac{\sqrt{2 \pi} \eta^{2}}{16 \varepsilon^{\frac{3}{2}} \lambda^{3} \rho^{2} g^{2} \delta} \operatorname{erfi}\left(\frac{\sqrt{2 \varepsilon} \rho g \delta \lambda}{\eta}\right)
\end{aligned}
$$

$$
\begin{aligned}
& U>-\beta \rho g \delta+\frac{\eta}{4 \rho g \varepsilon \lambda^{2}} e^{\left(\frac{2 \varepsilon \lambda^{2} \rho^{2} \delta^{2} \delta^{2}}{\eta^{2}}\right)} \\
& -\frac{\sqrt{2 \pi} \eta^{2}}{16 \varepsilon^{\frac{3}{2}} \lambda^{3} \rho^{2} g^{2} \delta} \operatorname{erfi}\left(\frac{\sqrt{2 \varepsilon} \rho g \delta \lambda}{\eta}\right)
\end{aligned}
$$

$$
\theta=\frac{1}{16 \rho^{2} g^{2} k \eta \varepsilon^{4}}\left[\begin{array}{l}
-2 \eta^{4}\left(e^{\frac{2 \rho^{2} g^{2} \varepsilon \varepsilon^{2}(x-\delta)^{2}}{\eta^{2}}}-e^{\frac{2 \rho^{2} g^{2} \varepsilon \varepsilon^{2} \delta^{2}}{\eta^{2}}}\right)+16 \theta_{0} \rho^{2} g^{2} k \eta \varepsilon \lambda^{4} \\
\rho g \sqrt{2 \pi} \varepsilon \lambda \eta^{3}\left\{(x-\delta) \operatorname{erfi}\left(\frac{\sqrt{2} \rho g \varepsilon \lambda(x-\delta)}{\eta}\right)-\operatorname{erfi}\left(\frac{\sqrt{2} \rho g \delta \delta \lambda}{\eta}\right)\right\}
\end{array}\right]
$$




\section{Results and Discussion}

We have extensively discussed steady thin film flow problem for lift, which is modeled by a Phan-Thein Tanner fluid for uniform thickness. The fluid is characterized as isothermal, steady incompressible. This leads to nonlinear differential equation. The exact solution, which gives velocity profile of fluid and temperature distribution, is obtained. The various parameters influence the behavior of velocity $v x$ and temperature distribution $\theta(x)$ of fluid. The relation of various parameters to velocity and temperature is investigated. The effects of the Slip parameter $\beta$, uniform thickness $\delta$, constant viscosity $\eta$, elongational behavior $\varepsilon$, relaxation time $\lambda$, constant density $\rho$ on velocity of fluid are presented in Figs. 2-7 respectively. The effect of the uniform thickness $\delta$, elongational behavior $\varepsilon$, constant density $\rho$, thermal conductivity $k$, relaxation time $\lambda$, constant viscosity $\eta$ on temperature distribution are shown in the Figs. 8-13. In the Fig. 2-7, one can observe that the parameters $\beta$ and $\eta$ are directly proportional with the magnitude of velocity; i.e. increase in these parameters causes increase in velocity. Further, the parameters $\delta$ $\varepsilon \lambda$ and $\rho$ are inversely proportional to the magnitude of velocity; i.e decrease in these parameters leads to the increase in velocity. In the Fig. 8-13, it can easily be perceived that the temperature profile decreases when the increase in parameter $k$ and $\lambda$. Also one can observe that an increase in parameters $\delta \varepsilon \eta$ and $\rho$ causes increase in temperature. The velocity profile of PTT fluid with velocity of fluid with the velocity of fluid for its special cases is thoroughly compared in Table 1, when certain parameters are fixed. Such mention can be comprehended from caption of Table. The numerically computed velocity at normal axis to the belt is presented in Table 1. The lower velocity of EPTT fluid, as compared to the velocity of its special cases is evident in Table 1. In the same pattern, Table 2 presents comparison of temperature distribution of PTT fluid with temperature distribution of its special cases. The caption of table indicates the parameters, which are kept fixed. Table also indicates that the temperature of EPTT fluid at various normal axis to the belt is higher than the temperature of fluid of its special cases.
Table 1. The velocity profile for various cases when $\eta=600$ poise $, \delta=1, \delta_{1}=1, \delta_{2}=1, \mathrm{U}=1$, $\beta=0.0001, \varepsilon=1.2, \lambda=50, \rho=0.65 \mathrm{~g} / \mathrm{cm}^{3}$

\begin{tabular}{|l|l|l|l|l|l|}
\hline$x$ & UCM & LPTT & QPTT & CPTT & EPTT \\
\hline 0 & 1.00064 & 1.00064 & 1.00064 & 1.00064 & 1.00064 \\
\hline 0.2 & 0.998726 & 0.997666 & 0.997368 & 0.997339 & 0.997301 \\
\hline 0.4 & 0.99724 & 0.995677 & 0.995292 & 0.995258 & 0.995214 \\
\hline 0.6 & 0.996178 & 0.994429 & 0.994026 & 0.993992 & 0.993947 \\
\hline 0.8 & 0.995541 & 0.993749 & 0.993344 & 0.99331 & 0.993265 \\
\hline 1 & 0.995329 & 0.993534 & 0.993129 & 0.993095 & 0.99305 \\
\hline
\end{tabular}

Table 2. The temperature distribution for various cases when $\eta=600$ poise, $\varepsilon=1.2, \lambda=50$, $\rho=0.9 \mathrm{~g} / \mathrm{cm}^{3}, \delta=1, \delta_{1}=1, \delta_{2}=1$, $\theta=1 K, \eta=450$ poise, $k=23 \mathrm{~W} /(m \cdot K)$

\begin{tabular}{|l|l|l|l|l|l|}
\hline$x$ & UCM & LPTT & QPTT & CPTT & EPTT \\
\hline 0 & 1 & 1 & 1 & 1 & 1 \\
\hline 0.2 & 1.00037 & 1.00115 & 1.00215 & 1.00302 & 1.00467 \\
\hline 0.4 & 1.00055 & 1.00155 & 1.00273 & 1.00377 & 1.00544 \\
\hline 0.6 & 1.00061 & 1.00166 & 1.00286 & 1.00391 & 1.00558 \\
\hline 0.8 & 1.00063 & 1.00168 & 1.00288 & 1.00393 & 1.0056 \\
\hline 1 & 1.00063 & 1.00169 & 1.00288 & 1.00393 & 1.0056 \\
\hline
\end{tabular}

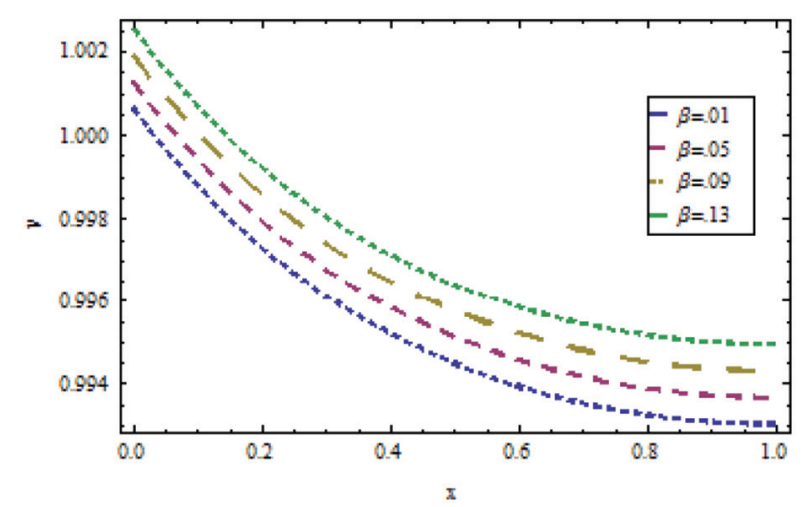

Figure 2. The velocity profile for different values of $\beta$, when $U=1, \eta=600$ poise, $\rho=0.65 \mathrm{~g} / \mathrm{cm}^{3}, \lambda=50$, $\varepsilon=1.2, \delta=1$ 


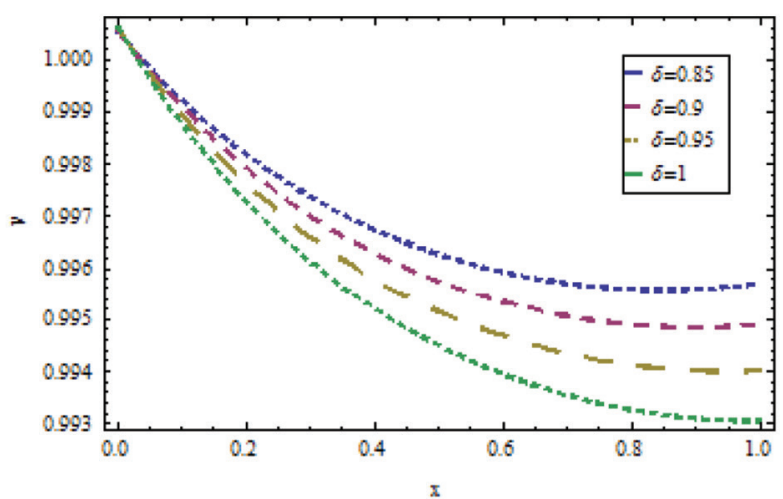

Figure 3. The velocity profile for different values of $\delta$, when $U=1, \eta=600$ poise, $\rho=0.65 \mathrm{~g} / \mathrm{cm}^{3}, \lambda=50$, $\varepsilon=1.2, \beta=0.0001$

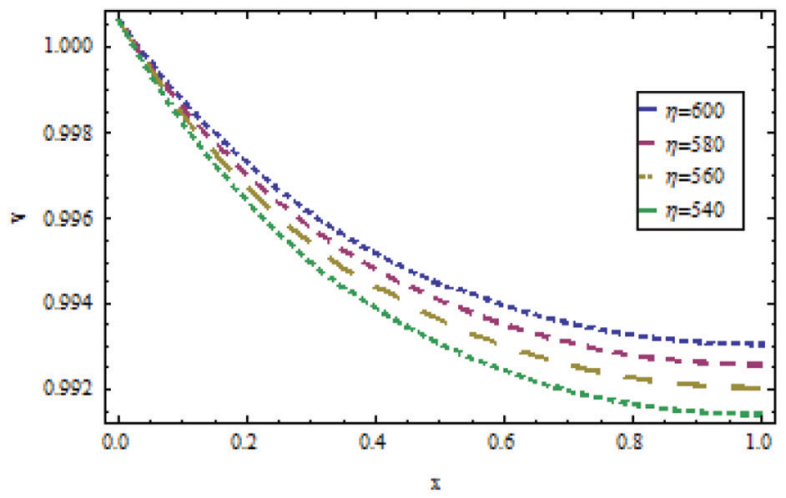

Figure 4. The velocity profile for different values of $\eta$, when $U=1, \rho=0.65 \mathrm{~g} / \mathrm{cm}^{3}, \beta=0.0001, \lambda=50$, $\varepsilon=1.2, \delta=1$

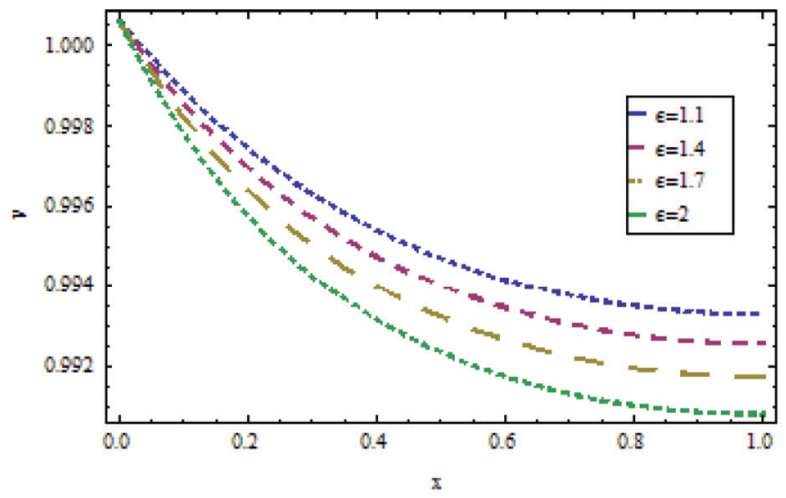

Figure 5. The velocity profile for different values of $\varepsilon$, when $U=1, \eta=600$ poise, $\rho=0.65 \mathrm{~g} / \mathrm{cm}^{3}, \lambda=50$, $\delta=1.2, \beta=0.0001$

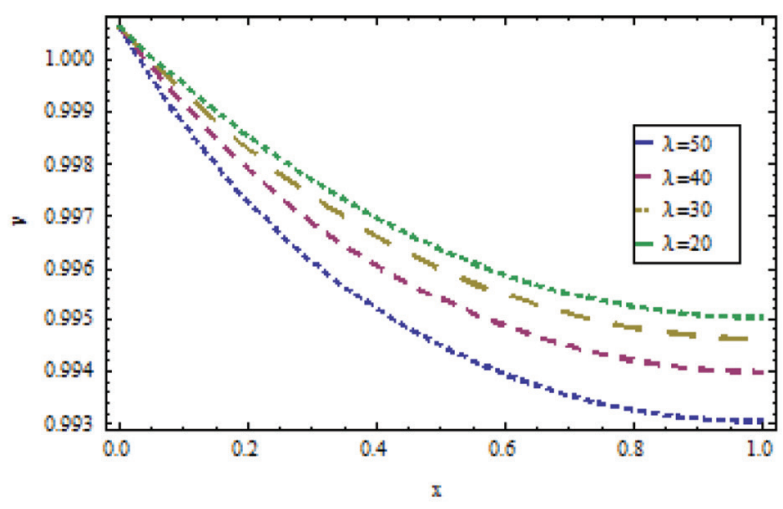

Figure 6. The velocity profile of different values of $\lambda$, when $U=1, \eta=600$ poise, $\rho=0.65 \mathrm{~g} / \mathrm{cm}^{3}, \varepsilon=1.2$, $\beta=0.0001, \delta=1$

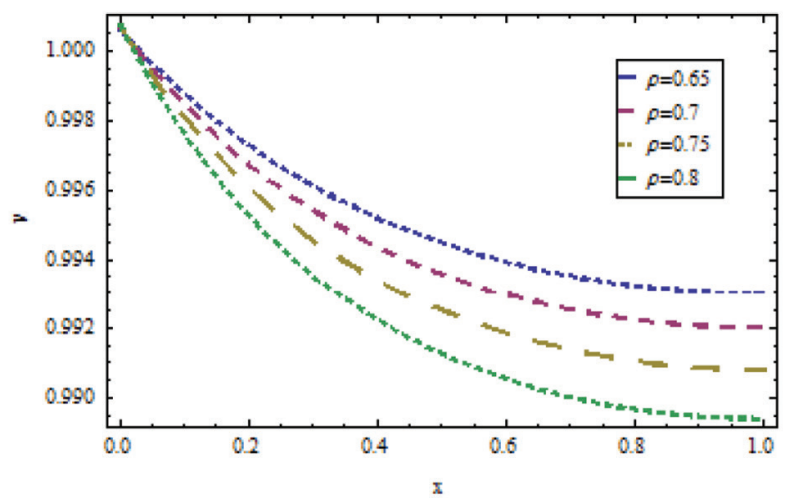

Figure 7. The velocity profile for different values of $\rho$, when $U=1, \eta=600$ poise, $\varepsilon=1.2, \beta=0.0001$, $\delta=1, \lambda=50$

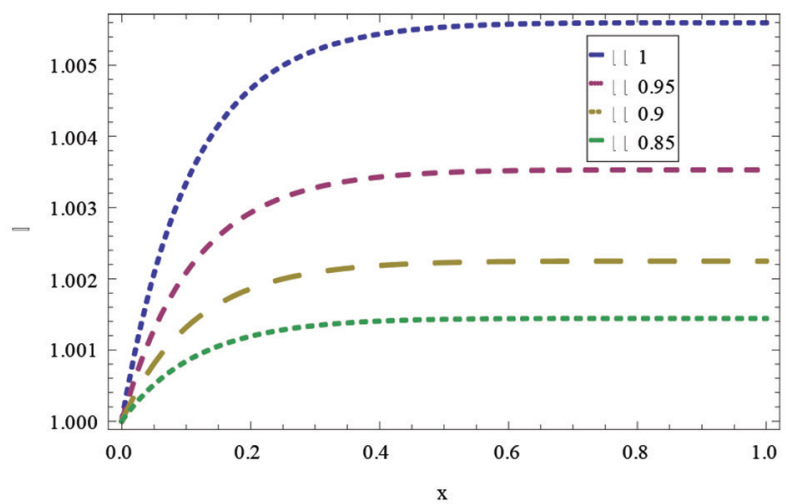

Figure 8. The temperature distribution for different $\delta$, when $\varepsilon=2.2, \lambda=50, \rho=0.9 \mathrm{~g} / \mathrm{cm}^{3}, \theta_{0}=1 K, \eta=$ 450 poise, $k=23 \mathrm{~W} /(m \cdot K)$ 


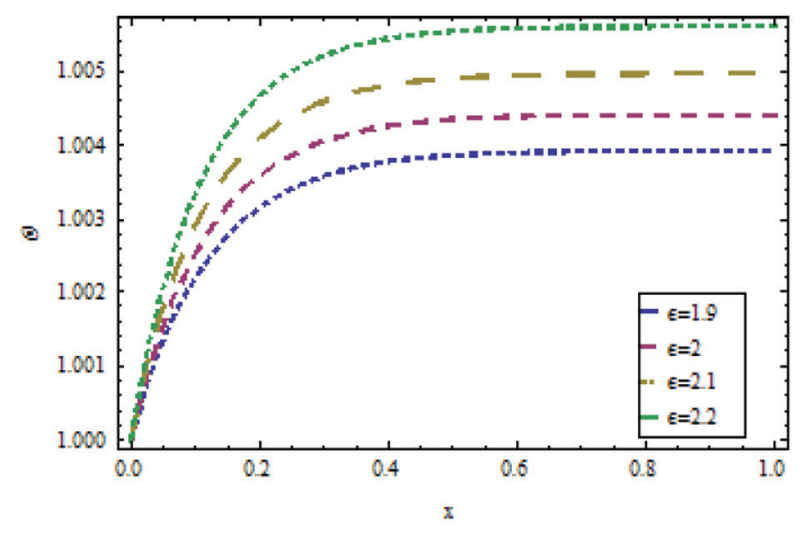

Figure 9. The temperature distribution for different values of the $\varepsilon$, when $\delta=1, \lambda=50, \rho=0.9 \mathrm{~g} / \mathrm{cm}^{3}, \theta_{0}=1 \mathrm{~K}$, $\eta=450$ poise, $k=23 \mathrm{~W} /(m \cdot K)$

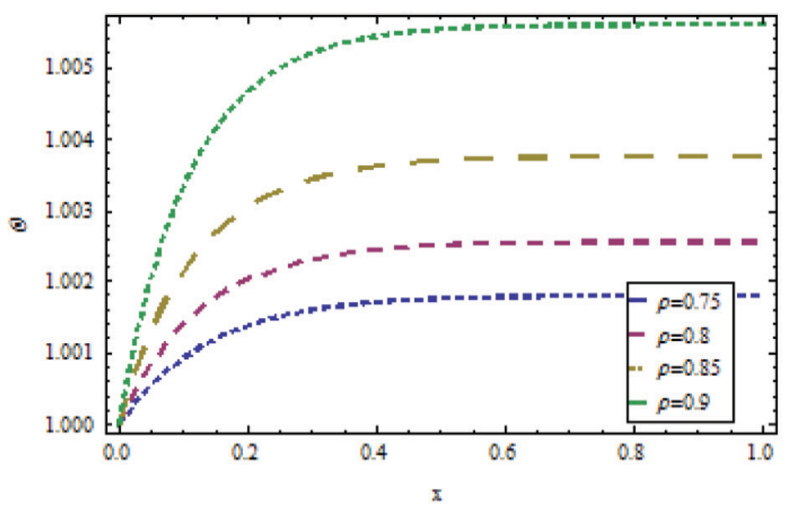

Figure 10. The temperature distribution for different $\rho$, when $\delta=1, \lambda=50, \varepsilon=2.2, \theta_{0}=1 K, \eta=450$ poise, $k=23 W /(m \cdot K)$

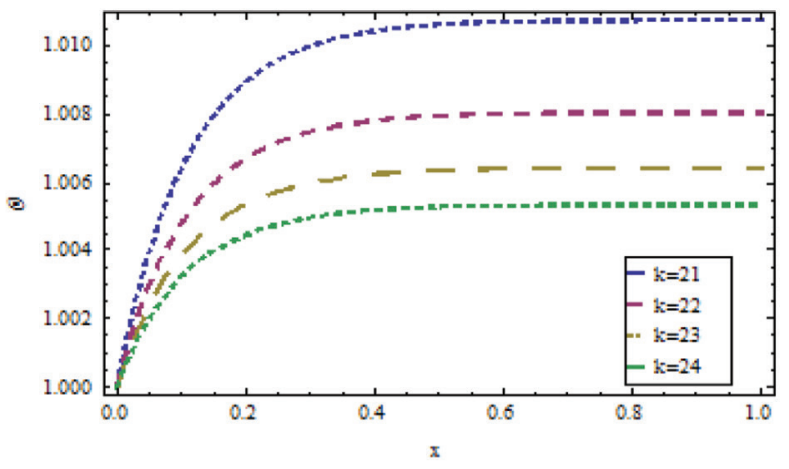

Figure 11. The temperature distribution for different values of $k$, when $\varepsilon=2.2, \lambda=50, \delta=1, \theta_{0}=1 \mathrm{~K}$, $\eta=450$ poise, $\rho=0.9 \mathrm{~g} / \mathrm{cm}^{3}$

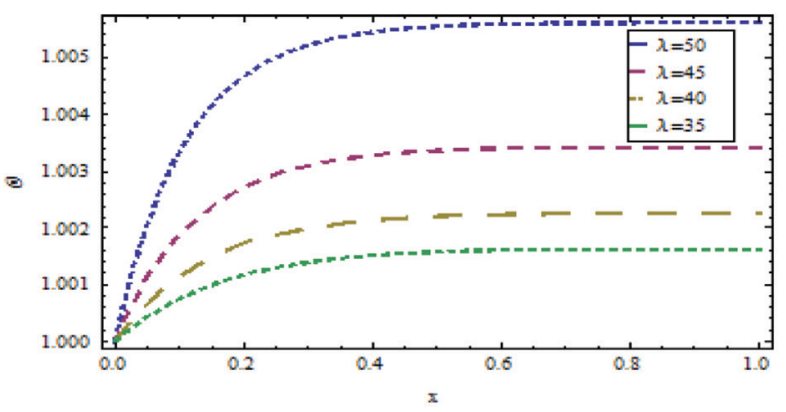

Figure 12. The temperature distribution for different values of $\lambda$, when $\varepsilon=2.2, \rho=0.9 \mathrm{~g} / \mathrm{cm}^{3}, \delta=1, \theta_{0}$ $=1 K, \eta=450$ poise, $k=23 \mathrm{~W} /(m \cdot K)$

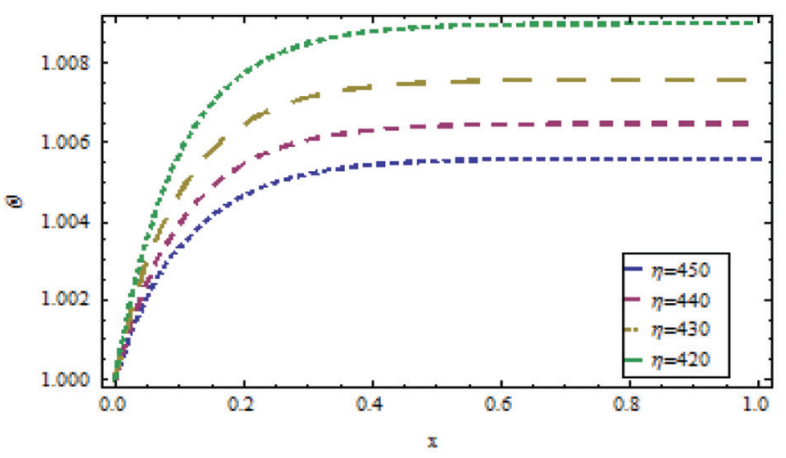

Figure 13. The temperature distribution for different values of $\eta$, when $\varepsilon=2.2, \rho=0.9 \mathrm{~g} / \mathrm{cm}^{3}, \delta=1, \theta_{0}$ $=1 K, \delta=1, k=23 \mathrm{~W} /(m \cdot K)$

\section{Conclusions}

We have obtained the exact solutions of the thin film flow using Phan-Thein Tanner fluid model, characterized as incompressible and isothermal thermal for uniform thickness on a vertical belt. We have also obtained solutions of the special cases such as UCM, LPTT, QPTT, CPTT and as well as EPTT. Here we have noted that if we increase the slip effect on a vertical belt then PTT fluid model will uplift quickly as compare to no slip, EPTT fluid model will flow against the direction of gravitation slowly as compare to CPTT, QPTT and UCM fluid model and for temperature distribution UCM fluid model has les temperature as compare to LPTT, QPTT, CPTT and EPTT.

\section{References}

1. Non-Newtonian Flow in the Process Industries [internet]. https://www.sciencedirect.com/book/9780750637701/ 
non-newtonian-flow-in-the-process-industries. Date accessed: 1999.

2. Memon KN, Khan SA, Islam S, Zafar NA, Shah SF, Siddiqui AM. Unsteady Drainage of Electrically Conducting Power Law Fluid. Applied Mathematics \& Information Sciences. 2014; 8 (5): 2287-2296. https://doi.org/10.12785/ amis/080523.

3. Dunn JE, Rajagopal KR. Fluids of differential type: Critical review and thermodynamic analysis. Int. J. Eng. Sci. 1995; 33(5): 689-729. https://doi.org/10.1016/00207225(94)00078-X.

4. Faraz N, Lei H, Khan Y. Analytical Solution of Linear, Quadratic and Cubic Model of PTT Fluid. JACM. 2015; 1(4): 220-228.

5. Memon KN, Shah SF, Siddiqui AM. Exact solution of unsteady tank drainage for Ellis Fluid. J APPL FLUID MECH. ISSN, 2018; 11(6): 1629-1636. https://doi. org/10.29252/jafm.11.06.28890.

6. Siddiqui AM, Walait A, Haroon T, Ashraf H. On the study of stationary points and uniform thickness of PTT fluid film on a vertically upward moving belt. Can. J. Phys. 2016; 94(10): 982-991. https://doi.org/10.1139/cjp-2014-0591.

7. Siddiqui AM, Mahmood R, Ghori QK. Some exact solutions for the thin film flow of a PTT fluid. Physics Letters A. 2006; 356(4-5): 353-356. https://doi.org/10.1016/j.physleta.2006.03.071.

8. Channer S, Memon KN, Ghoto AA, Siddiqui AM, Shah SF. Analytical Solutionof Lift for Thin Film Flow for Phan Thien Tanner Fluid. SIND UNIV RES J (SCI SER). 2019; 51(2): 215-222. https://doi.org/10.26692/sujo/2019.6.37.

9. Farooq M, Rahim MT, Islam S, Arif M. Series Solutions of Lifting and Drainage Problems of a Nonisothermal Modified
Second Grade Fluid Using a Vertical Cylinder. J. Appl. Math. 2014; 1-8. https://doi.org/10.1155/2014/842650.

10. Farooq M, Rahim MT, Islam S, Siddiqui AM. Steady Poiseuille flow and heat transfer of couple stress fluids between two parallel inclined plates with variable viscosity. J. Assoc. Arab Univ. Basic Appl. 2013; 14(1): 9-18. https:// doi.org/10.1016/j.jaubas.2013.01.004.

11. Bramble JH, Pasciak JE, Wang J, Xu J. Convergence estimates for multigrid algorithms without regularity assumptions. Mathematics of Computation. 1991; 57: 23-45. https://doi. org/10.1090/S0025-5718-1991-1079008-4.

12. Wang CY. Exact Solutions of the Unsteady Navier-Stokes Equations. Applied Mechanics Reviews. 1989; 42(11): 269282. https://doi.org/10.1115/1.3152400.

13. Benharbit AM, Siddiqui AM. Certain solutions of the equations of the planar motion of a second grade fluid for steady and unsteady cases. Acta Mechanica. 1992; 94(1-2): 85-96. https://doi.org/10.1007/BF01177007.

14. Siddiqui AM, Kaloni PN. Certain inverse solutions of a non-Newtonian fluid. INT J NONLIN MECH. 1986; 21(6): 459-473. https://doi.org/10.1016/0020-7462(86)90042-9.

15. Memon KN, Siddiqui A, Shah SF. Exact Solution of Tank Drainage for Newtonian fluid with Slip Condition. SURJ Sindh University Research Journal. 2017; 49(2) 283-288.

16. Siddiqui A, Ashraf A, Azim QA, Babcock B. Exact solutions for thin film flows of a PTT fluid down an inclined plane and on a vertically moving belt. Adv. Studies Theor. Phys. 2013; 7(2): 65-87. https://doi.org/10.12988/astp.2013.13005.

17. Dynamics of polymeric liquids [internet]. https://www. wiley.com/en-Dynamics+of+Polymeric+Liquids\% $2 \mathrm{C}+$ Volume $+1 \% 3 \mathrm{~A}+$ Fluid+Mechanics $\% 2 \mathrm{C}+2 \mathrm{nd}+$ Edition -p-9780471802457. Date accessed: 05/1987 